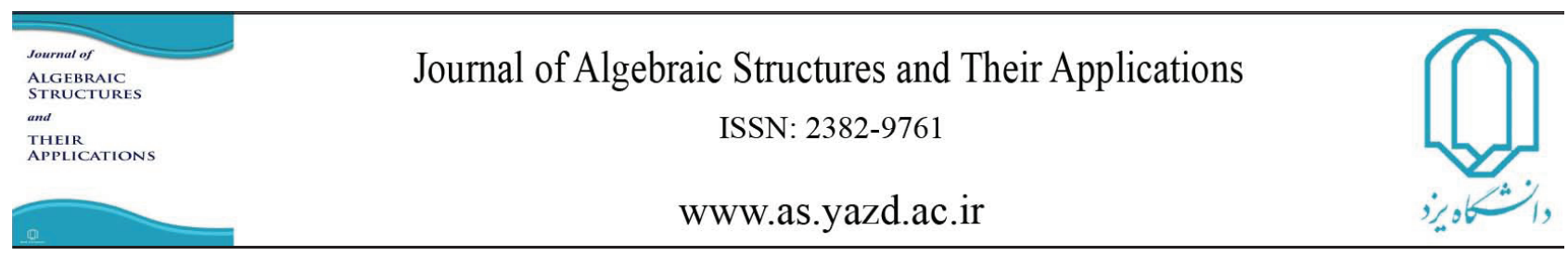

Algebraic Structures and Their Applications Vol. 4 No. 2 ( 2017 ) pp 1-14.

\title{
RESULTS ON ENGEL FUZZY SUBGROUPS
}

\author{
E. MOHAMMADZADEH, R.A. BORZOOEI ${ }^{(*)}$ AND Y.B. JUN
}

Communicated by B. Davvaz

\begin{abstract}
In the classical group theory there is an open question: Is every torsion free $n$ Engel group (for $n \geq 4$ ), nilpotent?. To answer the question, Traustason [II] showed that with some additional conditions all 4-Engel groups are locally nilpotent. Here, we gave some partial answer to this question on Engel fuzzy subgroups. We show that if $\mu$ is a normal 4-Engel fuzzy subgroup of group $G, x, y \in G$ and $a=y^{x}$, then $\left.\mu\right|_{<a, a^{y}>}$ is a generalized nilpotent of class at most 2. Also we define a torsion free fuzzy subgroup and show that if $\mu$ is a 4-Engel torsion free fuzzy subgroup of $G$, then $\left.\mu\right|_{<a, y>}$ is a generalized nilpotent of class at most 4 , for conjugate elements $a, y$ in $G$.
\end{abstract}

\section{INTRODUCTION}

Engel groups are certain generalized nilpotent groups which have received considerable attention in recent years. Nilpotent groups arise in Galois theory, as well as in the classification of groups. They also appear prominently in the classification of Lie groups. In mathematics, more specifically in abstract algebra, Galois theory, named after Evariste Galois, provides a DOI:http://dx.doi.org/10.29252/asta.4.2.1

MSC(2010): Primary: 20N25, 20F45, 20F18.

Keywords: $n$-Engel group, (torsion free) $n$-Engle fuzzy subgroup, generalized nilpotent fuzzy subgroup.

Received: 16 August 2017, Accepted: 28 April 2018

*Corresponding author

(C) 2017 Yazd University. 
connection between field theory and group theory. Using Galois theory, certain problems in field theory can be reduced to group theory, which is, in some sense, simpler and better understood. In 1936, Zorn [15] proved that each finite Engel group is nilpotent. Golod [4] has constructed finitely generated Engel group that are not nilpotent. There are 3-Engel 2-groups and 5-groups that are not nilpotent. Therefore, each Engel group is not nilpotent in general and so scientists study on conditions that make $n$-Engel groups, nilpotent. Also, it is interesting to know which subgroups of Engel groups are nilpotent. In Engel groups we have the following question: $\mathbf{Q}$ : Is every torsion free $n$-Engel group, nilpotent? The answer is positive for $n \leq 3$, but it remain open for $n \geq 4$. Moreover if $n=1$, then every 1-Engel group is Abelian. Levi [7] proved that 2-Engel groups are nilpotent of class at most 3. For 3-Engel groups the problem is much harder. Heineken in [5] has shown that every 3-Engel group $G$ is nilpotent of class at most 4 if $G$ has no element of order 2 or 5 . L.Kappe and W.Kappe [6] gave a characterization of 3-Engel groups which is analogous to Levi's theorem on 2-Engel groups. They showed that the following are equivalent:

(i) $G$ is a 3-Engel group. (ii) $x^{G}$ is a 2-Engel group for any $x \in G$. (iii) for any $x \in G$ we have that $x^{G}$ is nilpotent of class at most 2 .

Traustason [I] studied 4-Engel groups. He proved that with some additional conditions every $r$ generator subgroup of a 4-Engel group has nilpotency class at most $6 r$. Also he proved that if $G$ is a 4-Engel group, then $\left\langle a, a^{b}\right\rangle$ is nilpotent, where $a$ is of finite order and $b \in G$. Applying the concept of fuzzy sets of Zadeh [14] to group theory, Rosenfeld [10] introduced the notion of a fuzzy subgroup as early as 1971. With appropriate definitions in the fuzzy setting, most of the elementary results of group theory have been superseded with a startling generalized effect. In [3] we proposed a definition for nilpotent fuzzy subgroup through its ascending central series and called it g-nilpotent fuzzy subgroups. Also in [T] we studied $n$-Engel fuzzy subgroups for $n \leq 3$. Now in this paper, we centered on 4-Engel fuzzy subgroups. Specially, we investigate some conditions that 4-Engel fuzzy subgroups will be generalized nilpotent. Then we shall give some partial answer to the following question, which is fuzzy analogies of the main question on Engel groups.

Q : Is every torsion free $n$-Engel fuzzy subgroup, generalized nilpotent?

Moreover, we show that if $\mu$ is a 4-Engel torsion free fuzzy subgroup of a group $G$, then $\left.\mu\right|_{<a, y>}$ is generalized nilpotent of class at most 4 , for conjugate elements $a, y$ in $G$.

\section{Preliminaries}

Let $G$ be any group and $x, y \in G$. Define the $n$-commutator $\left[x,_{n} y\right]$, for any $n \in \mathbb{N}$ and $x, y \in G$, by $\left[x,_{0} y\right]=x,\left[x,_{1} y\right]=x^{-1} y^{-1} x y$ and $\left[x,_{n} y\right]=\left[\left[x,_{n-1} y\right], y\right]$. Now a group $G$ is 
called an Engel group if for each ordered pair $(x, y)$ of elements in $G$ there is a positive integer $n=n(x, y)$, such that $[x, n y]=e$, for any $x, g \in G$ and $n \in \mathbb{N}$. If $n=n(x, y)$ is chosen independently of $x, y$, then we say that $G$ is an $n$-Engel group (See $[\bar{G}]$ ).

Definition 2.1. [9] Let $X_{1}, X_{2}, \ldots$ be nonempty subsets of a group $G$. Define the commutator subgroup of $X_{1}$ and $X_{2}$ by

$$
\left[X_{1}, X_{2}\right]=\left\langle\left[x_{1}, x_{2}\right] \mid x_{1} \in X_{1}, x_{2} \in X_{2}\right\rangle .
$$

More generally, define

$$
\left[X_{1}, \ldots, X_{n}\right]=\left[\left[X_{1}, \ldots, X_{n-1}\right], X_{n}\right]
$$

where $n \geq 2$ and $\left[X_{1}\right]=\left\langle X_{1}\right\rangle$. Also recall that $X_{1}^{X_{2}}=\left\langle x_{1}^{x_{2}} \mid x_{1} \in X_{1}, x_{2} \in X_{2}\right\rangle$, where $x_{1}^{x_{2}}=x_{2}^{-1} x_{1} x_{2}$.

Definition 2.2. A group $G$ is called torsion free if no element of $G$ has finite order than 0 . Also two element $a, y$ in $G$ are conjugate if $a=y^{x}$ for some $x \in G$.

Example 2.3. Let $S_{3}=\left\langle a, b \mid a^{3}=b^{2}=e, a^{b}=a^{-1}\right\rangle$. Then $a$ and $a^{-1}$ are conjugate elements.

Theorem 2.4. [9] Let $G$ be a group and $x, y, z \in G$. Then

(i) $[x, y]=[y, x]^{-1}$,

(ii) $[x . y, z]=[x, z]^{y} \cdot[y, z]$ and $[x, y . z]=[x, z] \cdot[x, y]^{z}$,

(iii) $\left[x, y^{-1}\right]=\left([x, y]^{y^{-1}}\right)^{-1}$ and $\left[x^{-1}, y\right]=\left([x, y]^{x^{-1}}\right)^{-1}$.

Note that $x^{g}=x \cdot[x, g]$.

Definition 2.5. [14] A fuzzy subset of $X$ is a function from $X$ into [0,1].

Definition 2.6. [8] Let $\mu$ be a fuzzy subset of a group $G$. Then $\mu$ is called a fuzzy subgroup of $G$ if for all $x, y \in G ; \mu(x y) \geq \mu(x) \wedge \mu(y)$, and $\mu\left(x^{-1}\right) \geq \mu(x)$. A fuzzy subgroup $\mu$ of $G$ is called normal if $\mu(x y)=\mu(y x)$, for all $x, y$ in $G$.

Theorem 2.7. [8] Fuzzy subgroup $\mu$ of $G$ is normal if and only if $\mu_{t}=\{x \in G: \mu(x) \leq t\}$ is a normal subgroup of $G$ for any $t \in \mu(G) \bigcup\{b \in[0,1] \mid b \leq \mu(e)\}$ if and only if $\mu(x)=\mu\left(y^{-1} x y\right)$, for any $x, y \in G$.

Theorem 2.8. [8] Let $\mu$ be a fuzzy subgroup of $G$.

(i) If $\mu\left(x y^{-1}\right)=\mu(e)$, then $\mu(x)=\mu(y)$.

(ii) For any $x, y \in G, \mu(x) \neq \mu(y)$, implies $\mu(x y)=\mu(x) \wedge \mu(y)$.

(iii) If $H$ is a subgroup of $G$, then $\left.\mu\right|_{H}$, where $\left.\mu\right|_{H}: H \longrightarrow[0,1]$ is defined by

$\left.\mu\right|_{H}(x)=\mu(x)$ for any $x \in H$, is a fuzzy subgroup of $H$.

Definition 2.9. [8] Let $\mu$ be a fuzzy subset of a semigroup $G$ and 


$$
Z(\mu)=\{x \in G \mid \mu(x y)=\mu(y x) \text { and } \mu(x y z)=\mu(y x z), \text { for any } y, z \in G\} .
$$

If $Z(\mu)=G$, then $\mu$ is called commutative in $G$.

Theorem 2.10. [8] Let $\mu$ be a fuzzy subset of a semigroup $G$. If $Z(\mu)$ is nonempty, then $Z(\mu)$ is a subsemigroup of $G$. Moreover, if $G$ is a group, then $Z(\mu)$ is a normal subgroup of $G$. Also, if $T=\left\{x \in G \mid \mu\left(x y x^{-1} y^{-1}\right)=\mu(e)\right.$, for any $\left.y \in G\right\}$, then $T=Z(\mu)$.

Notation . From now on, we let $G$ be a group unless otherwise specified.

We recall the notion of the ascending central series of a fuzzy subgroup of $G$ [3]. Let $\mu$ be a fuzzy subgroup of $G$. Put $Z_{0}(\mu)=\{e\}$. Clearly $Z_{0}(\mu) \unlhd G$. Let $Z_{1}(\mu)=\{x \in G \mid \mu([x, y])=$ $\mu(e)$, for any $y \in G\}$. Now using Theorem [2.10, we have $Z_{1}(\mu)=Z(\mu)$ is a normal subgroup of $G$. We define a subgroup $Z_{2}(\mu)$ of $G$ such that $\frac{Z_{2}(\mu)}{Z_{1}(\mu)}=Z\left(\frac{G}{Z_{1}(\mu)}\right)$; since $Z_{1}(\mu) \unlhd G$ then $Z_{1}(\mu) \unlhd Z_{2}(\mu)$. We show that $\left[Z_{2}(\mu), G\right] \subseteq Z_{1}(\mu)$. For this, let $x \in Z_{2}(\mu)$ and $g \in G$. Thus $x Z_{1}(\mu) \in \frac{Z_{2}(\mu)}{Z_{1}(\mu)}=Z\left(\frac{G}{Z_{1}(\mu)}\right)$, which implies that $\left[x Z_{1}(\mu), g Z_{1}(\mu)\right]=Z_{1}(\mu)$ for any $g \in G$. Hence $[x, g] \in Z_{1}(\mu)$ and so $\left[Z_{2}(\mu), G\right] \subseteq Z_{1}(\mu)$. Thus $x^{g}=x[x, g] \in Z_{2}(\mu)$. Therefore, $Z_{2}(\mu) \unlhd G$.

Similarly for $k \geq 2$, we define a normal subgroup $Z_{k}(\mu)$ such that $\frac{Z_{k}(\mu)}{Z_{k-1}(\mu)}=Z\left(\frac{G}{Z_{k-1}(\mu)}\right)$. The ascending normal series of $\mu$ is $Z_{0}(\mu) \subseteq Z_{1}(\mu) \subseteq Z_{2}(\mu) \subseteq \ldots$.

Lemma 2.11. [3] Let $\mu$ be a fuzzy subgroup of $G$. Then for $k \in \mathbb{N}$

$$
Z_{k}(\mu)=\left\{x \in G \mid \mu\left(\left[x, y_{1}, \ldots, y_{k}\right]\right)=\mu(e), \text { for any } y_{1}, y_{2}, \ldots, y_{k} \in G\right\} .
$$

Definition 2.12. [3] A fuzzy subgroup $\mu$ of $G$ is called a generalized nilpotent or briefly $g$ nilpotent if there exist none negative integer $n$, such that $Z_{n}(\mu)=G$. The smallest such integer is called the class of $\mu$.

\section{Results on Engel fuzzy subgroups}

In this section, first we define the notion of a torsion free fuzzy subgroup of $G$. Then we study g-nilpotency of specific fuzzy subgroups. Specially, we have centered mainly on the question weather torsion free $n$-Engel fuzzy subgroups are g-nilpotent. Moreover, we show that with some conditions, $\left.\mu\right|_{<a, y>}$ is a g-nilpotent of class at most 4 , where $\mu$ is a normal torsion free 4-Engel fuzzy subgroup of $G$ and $a, y$ are conjugate elements in $G$.

Definition 3.1. [I] A fuzzy subgroup $\mu$ of $G$ is called an $n$-Engel fuzzy subgroup if there exist a positive integer $n$, such that $\mu([x, n y])=\mu(e)$ for any $x, y \in G$. 
Example 3.2. Let $D_{3}=\left\langle a, b ; \quad a^{3}=b^{2}=e, \quad b a=a^{2} b\right\rangle$ be the dihedral group with six elements and $t_{0}, t_{1} \in[0,1]$ such that $t_{0}>t_{1}$. Define a fuzzy subgroup $\mu$ of $D_{3}$ as follows:

$$
\mu(x)=\left\{\begin{array}{lll}
t_{0} & \text { if } & x \in<a> \\
t_{1} & \text { if } & x \notin<a>
\end{array}\right.
$$

Then $\left(D_{3} \backslash\langle a\rangle\right)\left(D_{3} \backslash\langle a\rangle\right)=\langle a\rangle,(\langle a\rangle)\left(D_{3} \backslash\langle a\rangle\right)=\left(D_{3} \backslash\langle a\rangle\right),\left(D_{3} \backslash\langle a\rangle\right)(\langle a\rangle)=\left(D_{3} \backslash\langle a\rangle\right)$ and $(\langle a\rangle)(\langle a\rangle)=(\langle a\rangle)$. Now, we show that $\mu$ is 1-Engel. If $x \in\langle a\rangle$ and $y \notin\langle a\rangle$, then $x y \notin\langle a\rangle$. Thus by the above relations, we have $[x, y]=x^{-1} y^{-1} x y=(y x)^{-1}(x y) \in\langle a\rangle$, which implies that $\mu[x, y]=t_{0}=\mu(e)$. Similarly, for the cases $x \notin\langle a\rangle$ and $y \in\langle a\rangle$ or $x, y \in\langle a\rangle$ or $x, y \notin\langle a\rangle$, we have $\mu[x, y]=\mu(e)$. Hence for any $x, y \in D_{3}, \mu[x, y]=\mu(e)$ and so $\mu$ is 1-Engel. Note that, $D_{3}$ is not an Engel group, since $\left[a,_{n} b\right]=a \neq e$.

Definition 3.3. Let $\mu$ be a fuzzy subgroup of $G$. Then $\mu$ is called a torsion free fuzzy subgroup of $G$, if for some $s \in \mathbb{N}, \mu\left(x^{s}\right)=\mu(e)$ implies $\mu(x)=\mu(e)$.

Example 3.4. Let $G$ be a torsion free $n$-Engel group and $\mu: G \longrightarrow[0,1]$ is defined by:

$$
\mu(x)=\left\{\begin{array}{lc}
1, & x=e \\
1 / 2, & \text { otherwise }
\end{array}\right.
$$

for any $x \in G$. Then for any $t \in[0,1], \mu_{t}=\{x \mid \mu(x) \geq t\}$ is a normal subgroup of $G$ and so by Theorem [2.], $\mu$ is a normal fuzzy subgroup. Now, let $\mu\left(x^{n}\right)=\mu(e)$, for $x \in G$. Then $x^{n}=e$. Since $G$ is torsion free, $x=e$ which implies that $\mu(x)=\mu(e)$. Thus $\mu$ is torsion free. Since $G$ is $n$-Engel, $\mu$ is $n$-Engel. Therefore, $\mu$ is a normal torsion free n-Engel fuzzy subgroup of $G$.

Note. In Example [3.4, if $G$ is a non-Abelian torsion free $n$-Engel group and $n \geq 3$, then $\mu$ is a normal torsion free $n$-Engel fuzzy subgroup such that $\mu[x, y] \neq \mu(e)$. In the following we prove that with an additional condition for some $x, y \in G$ we have a normal torsion free $n$-Engel fuzzy subgroup $\mu$, such that $\mu[x, y]=\mu(e)$.

Lemma 3.5. Let $\mu$ be a normal torsion free $n$-Engel fuzzy subgroup of $G$. If $\mu\left[x^{s}, y\right]=\mu(e)$, for some positive integer $s \geq 1$ and $x, y \in G$, then $\mu[x, y]=\mu(e)$.

Proof. Let $\mu$ be a normal torsion free n-Engel fuzzy subgroup of $G$ and $i>1$ be the minimum positive integer such that $\mu\left[y,{ }_{i} x\right]=\mu(e)$. We show that

$$
\mu\left[y,{ }_{i-2} x, x^{s}\right]=\mu(e) . \quad(*)
$$


For this, we prove it by induction on $i \leq 2$. If $i=2$, then the result is immediate. Now let $\mu\left[y,{ }_{i-2} x, x^{s}\right]=\mu(e)$, for $i>2$. Then by Theorems [2.6 and 2.4 and since $\mu$ is normal, we have

$$
\begin{aligned}
\mu\left[y,{ }_{(i+1)-2} x, x^{s}\right] & =\mu\left[\left[\left[y,{ }_{(i-2)} x\right], x\right], x^{s}\right] \\
& =\mu\left[\left[y,{ }_{(i-2)} x\right]^{-1}\left[y,{ }_{(i-2)} x\right]^{x}, x^{s}\right] \\
& =\mu\left(\left[\left[y,,_{(i-2)} x\right]^{-1}, x^{s}\right]^{\left[y,{ }_{(i-2)}\right.}\right]^{x}\left[\left[y,{ }_{(i-2)} x\right]^{x}, x^{s}\right] \\
& \geq \mu\left(\left[\left[y,{ }_{(i-2)} x\right]^{-1}, x^{s}\right]^{\left[y,{ }_{(i-2)} x\right]^{x}} \wedge \mu\left[\left[y,{ }_{(i-2)} x\right]^{x}, x^{s}\right]\right. \\
& =\mu\left[\left[y,{ }_{(i-2)} x\right], x^{s}\right] \wedge \mu\left[\left[y,{ }_{(i-2)} x\right], x^{s}\right] \\
& =\mu(e)) .
\end{aligned}
$$

Hence we have $(*)$. If $\mu\left(\left[y,{ }_{i-1} x\right]^{s}\right)=\mu(e)$, since $\mu$ is torsion free, then $\mu\left(\left[y,{ }_{i-1} x\right]\right)=\mu(e)$. This gives a contradiction to the minimality of i. For the case $\mu\left(\left[y,{ }_{i-1} x\right]^{s}\right) \neq \mu(e)$, first we show that

$$
\mu\left(\left[\left[y,{ }_{i-2} x\right], x^{s}\right]\right)=\mu\left(\left[y,{ }_{i-1} x\right]^{s}[y, i x]\left[y,{ }_{i+1} x\right] \ldots\right) \quad(* *)
$$

Let $s=2$. Then by Theorem [2.4, we have

$$
\begin{aligned}
\mu\left[y,{ }_{(i-2)} x, x^{2}\right] & =\mu\left(\left[y,_{(i-2)} x, x\right]\left[y,{ }_{(i-2)} x, x\right]^{x}\right) \\
& =\mu\left(\left[y,{ }_{(i-1)} x\right]\left[y,{ }_{(i-1)} x\right]\left[y,{ }_{(i-1)} x, x\right]\right) \\
& =\mu\left(\left[y,_{(i-1)} x\right]^{2}\left[y,{ }_{(i)} x\right]\right) .
\end{aligned}
$$

By similar method we get $\left({ }^{*}\right)$ for $s \geq 2$. Also by $\mu\left(\left[y,{ }_{i+1} x\right]\right) \geq \mu\left(\left[y,{ }_{i} x\right]\right)=\mu(e)$, we get $\mu\left(\left[y,{ }_{i} x\right]\left[y,{ }_{i+1} x\right] \ldots\right)=\mu(e)$ and so by $(*),(* *)$ and Theorem [2.8(ii), we have

$$
\begin{aligned}
\mu(e) & =\mu\left[\left[y,{ }_{i-2} x\right], x^{s}\right]=\mu\left(\left[y,{ }_{i-1} x\right]^{s}\left[y,{ }_{i} x\right]\left[y,{ }_{i+1} x\right] \ldots\right)=\mu\left(\left[y,{ }_{i-1} x\right]^{s}\right) \wedge \mu\left(\left[y,{ }_{i} x\right]\left[y,{ }_{i+1} x\right] \ldots\right) \\
& =\mu\left(\left[y,{ }_{i-1} x\right]^{s}\right)
\end{aligned}
$$

which is a contradiction. This complete the proof. $\square$

Lemma 3.6. Let $G$ be a torsion free $n$-Engel group, $x, y \in G$ and there exists $s \in \mathbb{N}$ such that $\left[y, x^{s}\right]=e$. Then $[y, x]=e$.

Proof. Let there exists $s \in \mathbb{N}$ such that $\left[y, x^{s}\right]=e$, but $[y, x] \neq e$. Suppose that $i>1$ is the minimal positive integer such that $[y, i x]=e$. First we show that $\left[\left[y,{ }_{i-2} x\right], x^{s}\right]=e$, for any $i \geq 2$. For $i=2$ the proof is clear. Let $\left[\left[y,{ }_{i-2} x\right], x^{s}\right]=e$, for $i>1$. Then by Theorem [2.4, we 
have

$$
\begin{aligned}
{\left[y,{ }_{(i+1)-2} x, x^{s}\right] } & =\left[\left[\left[y,{ }_{(i-2)} x\right], x\right], x^{s}\right] \\
& =\left[\left[y,{ }_{(i-2)} x\right]^{-1}\left[y,{ }_{(i-2)} x\right]^{x}, x^{s}\right] \\
& =\left(\left[\left[y,{ }_{(i-2)} x\right]^{-1}, x^{s}\right]^{\left[y,{ }_{(i-2)} x\right]^{x}}\left[\left[y,{ }_{(i-2)} x\right]^{x}, x^{s}\right]\right. \\
& =e .
\end{aligned}
$$

Hence $\left[\left[y,{ }_{i-2} x\right], x^{s}\right]=e$ for any $i \geq 2$. Now, since $\left[\left[y,{ }_{i-2} x\right], x^{s}\right]=\left[y_{, i-1} x\right]^{s}\left[y,{ }_{i} x\right]\left[y,{ }_{i+1} x\right]$, we have $e=\left[\left[y,{ }_{i-2} x\right], x^{s}\right]=\left[y,{ }_{i-1} x\right]^{s}\left[y,{ }_{i} x\right]\left[y,{ }_{i+1} x\right]$. Since $\left[y,{ }_{i+1} x\right]=\left[y,{ }_{i} x\right]=e$, we get $e=\left[y,{ }_{i-1} x\right]^{s}$. Now by the hypotheses we have $\left[y_{, i-1} x\right]=e$, which contradicts the minimality of $i$.

Theorem 3.7. Let $\mu$ be a normal torsion free $n$-Engel fuzzy subgroup of $G, x, y \in G$ and $s \in \mathbb{N}$. If $\left.\mu\right|_{\left\langle x^{s}, y\right\rangle}$ is a g-nilpotent of class 2, then $\left.\mu\right|_{\langle x, y\rangle}$ is a g-nilpotent of class 2, too.

Proof. Let $x, y \in G$ and $s \in \mathbb{N}$. First we show that,

$$
Z\left(\left.\mu\right|_{\left\langle x^{s}, y\right\rangle}\right)=Z\left(\left.\mu\right|_{\langle x, y\rangle}\right) \cap\left\langle x^{s}, y\right\rangle
$$

Suppose that $w \in Z\left(\left.\mu\right|_{\left\langle x^{s}, y\right\rangle}\right)$. Then by Theorem [2.10, we have $\mu\left[x^{s}, w\right]=\mu(e)$ and so by Lemma [.5, $\mu[x, w]=\mu(e)$ which implies that $w \in Z\left(\left.\mu\right|_{\langle x, y\rangle}\right) \cap\left\langle x^{s}, y\right\rangle$. Hence $Z\left(\left.\mu\right|_{\left\langle x^{s}, y\right\rangle}\right) \subseteq$ $Z\left(\left.\mu\right|_{\langle x, y\rangle}\right) \cap\left\langle x^{s}, y\right\rangle$. Now let $w \in Z\left(\left.\mu\right|_{\langle x, y\rangle}\right) \cap\left\langle x^{s}, y\right\rangle$. Then $w \in\left\langle x^{s}, y\right\rangle, \mu[x, w]=\mu(e)$ and $\mu[y, w]=\mu(e)$ and so by Theorem [2.4,

$$
\mu\left[x^{s}, w\right]=\mu\left([x, w]^{x^{s-1}}\left[x^{s-1}, w\right]\right) \geq \mu\left([x, w]^{x^{s-1}}\right) \wedge \mu\left(\left[x^{s-1}, w\right]\right)=\mu\left(\left[x^{s-1}, w\right]\right)
$$

which implies that $\mu\left[x^{s}, w\right]=\mu\left[x^{s-1}, w\right]=\ldots=\mu[x, w]=\mu(e)$. Hence $Z\left(\left.\mu\right|_{\left\langle x^{s}, y\right\rangle}\right) \supseteq Z\left(\left.\mu\right|_{\langle x, y\rangle}\right.$ )$\cap\left\langle x^{s}, y\right\rangle$. Therefore $Z\left(\left.\mu\right|_{\left\langle x^{s}, y\right\rangle}\right)=Z\left(\left.\mu\right|_{\langle x, y\rangle}\right) \cap\left\langle x^{s}, y\right\rangle$. Also since $\left.\mu\right|_{\left\langle x^{s}, y\right\rangle}$ is g-nilpotent of class 2 , then by Lemma $\left[\mathbf{L}\right.$, we have $\mu\left[x^{s}, y, z\right]=\mu(e)$ for any $z \in\left\langle x^{s}, y\right\rangle$. Therefore by Theorem [2.10 and $\left(^{*}\right),\left[x^{s}, y\right] \in Z\left(\left.\mu\right|_{\langle x, y\rangle}\right)$. Thus

$$
\left[x^{s} Z\left(\left.\mu\right|_{\langle x, y\rangle}\right), y Z\left(\left.\mu\right|_{\langle x, y\rangle}\right)\right]=Z\left(\left.\mu\right|_{\langle x, y\rangle}\right)=1_{\frac{\langle x, y\rangle}{Z\left(\left.\mu\right|_{\langle x, y\rangle}\right)}} .
$$

Now we show that $\frac{\langle x, y\rangle}{Z\left(\left.\mu\right|_{\langle x, y\rangle}\right)}$ is a torsion free group. For this, let $a Z\left(\left.\mu\right|_{\langle x, y\rangle}\right) \in \frac{\langle x, y\rangle}{Z\left(\left.\mu\right|_{\langle x, y\rangle}\right)}$, where $a \in\langle x, y\rangle$ and for some $k \in \mathbb{N}, a^{k} \in Z\left(\left.\mu\right|_{\langle x, y\rangle}\right)$. Then we have $\mu\left[a^{k}, b\right]=\mu(e)$ for any $b \in\langle x, y\rangle$

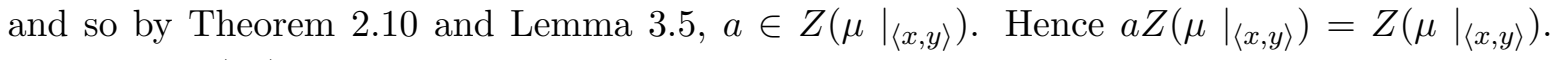
Therefore $\frac{\langle x, y\rangle}{Z\left(\left.\mu\right|_{\langle x, y\rangle}\right)}$ is a torsion free group. Since $\mu$ is a normal $n$-Engel fuzzy subgroup so for any $z \in\langle x, y\rangle, \mu\left[\left[x,_{n} y\right], z\right] \geq \mu\left[x,_{n} y\right]=\mu(e)$ and so by Theorem [2.10, $\left[x,_{n} y\right] \in Z\left(\left.\mu\right|_{\langle x, y\rangle}\right)$ which implies that

$$
\left[x Z\left(\left.\mu\right|_{\langle x, y\rangle}\right)_{, n} y Z\left(\left.\mu\right|_{\langle x, y\rangle}\right)\right]=Z\left(\left.\mu\right|_{\langle x, y\rangle}\right)
$$


Then $\frac{\langle x, y\rangle}{Z\left(\left.\mu\right|_{\langle x, y\rangle}\right)}$ is $n$-Engel and so by Lemma [3.6), we have $\left[x Z\left(\left.\mu\right|_{\langle x, y\rangle}\right), y Z\left(\left.\mu\right|_{\langle x, y\rangle}\right)\right]=1$. Hence $[x, y] \in Z\left(\left.\mu\right|_{\langle x, y\rangle}\right)$. Therefore, by Theorem [2.10 and Lemma [2.], $\left.\mu\right|_{\langle x, y\rangle}$ is a g-nilpotent of class at most 2 , too.

Theorem 3.8. Let $\mu$ be a normal 4-Engel fuzzy subgroup of $G, x, y \in G$ and $a=y^{x}$. Then $\left.\mu\right|_{<a, a^{y}>}$ is a g-nilpotent of class at most 2 .

Proof. Since $\mu$ is a 4-Engel fuzzy subgroup of $G$, for any $y \in G, \mu\left[x^{-1}, y, y, y, y\right]=\mu(e)$. Now Using Theorem [2.4 and $\mu$ is normal, we have

$$
\mu(e)=\mu\left[\left[x^{-1}, y\right], y, y, y\right]=\mu\left[[x, y]^{-x^{-1}},{ }_{3} y\right]=\mu\left[y, x,{ }_{3} y^{x}\right]^{x^{-1}}=\mu\left[y, x,{ }_{3} y^{x}\right]=\mu\left[y^{-1} y^{x},{ }_{3} y^{x}\right]
$$

which implies that $\mu(e)=\mu\left(\left[y^{-1}, 3 y^{x}\right]\right)$. By putting $a=y^{x}$ we have $\mu\left[y^{-1}, a, a, a\right]=\mu(e)$. Now, by Theorem $[2.4$ and since $\mu$ is normal, we get

$\mu(e)=\mu\left[y^{-1},{ }_{3} a\right]=\mu\left[[a, y]^{y^{-1}, 2} a\right]=\mu\left[a, y, 2 a^{y}\right]^{y^{-1}}=\mu\left[a, y,{ }_{2} a^{y}\right]=\mu\left[a^{-1} a^{y}{ }_{2} a^{y}\right]=\mu\left[a^{-1}, 2 a^{y}\right]$.

Therefore $\mu\left[a^{-1}, a^{y}, a^{y}\right]=\mu(e) \quad(*)$. Now, by puting $y^{-1}$ instead of $y$ and $a^{-1}$ instead of $a$ in $\left(^{*}\right)$ we have $\mu\left[a,\left(a^{-1}\right)^{y^{-1}},\left(a^{-1}\right)^{y^{-1}}\right]=\mu(e)$ and so by conjugacy we have $\mu\left[a^{y}, a^{-1}, a^{-1}\right]=\mu(e)$. Hence by Theorem [2.4,

$$
\begin{aligned}
\mu(e) & =\mu\left[a^{y}, a^{-1}, a^{-1}\right]=\mu\left[\left[a^{y}, a\right]^{-a^{-1}}, a^{-1}\right] \\
& =\mu\left[\left[a^{y}, a\right]^{-1}, a^{-1}\right]^{a^{-1}}=\mu\left[\left[a^{y}, a\right], a^{-1}\right]^{-\left[a^{y}, a\right]^{-1} a^{-1}}=\mu\left[\left[a^{y}, a\right], a^{-1}\right] \\
& =\mu\left[\left[a^{y}, a\right], a\right] .
\end{aligned}
$$

Similarly,

$$
\mu\left[a, a^{y}, a\right]=\mu(e), \quad \mu\left[a, a^{y}, a^{y}\right]=\mu(e) .
$$

Therefore by Lemma $\left[. \amalg,\left.\mu\right|_{<a, a^{y}>}\right.$ is g-nilpotent of class at most 2 .

Theorem 3.9. Let $\mu$ be a normal torsion free 4-Engel fuzzy subgroup of $G$ and $a, y$ be conjugate elements of $G$. Then $\left.\mu\right|_{<a, a^{y}>},\left.\mu\right|_{<a, a^{y^{2}}>},\left.\mu\right|_{<y, y^{a}>},\left.\mu\right|_{<y, y^{a^{3}}>}$ and $\left.\mu\right|_{<a, a^{y^{3}}>}$ are g-nilpotent of class at most 2.

Proof. By Theorem [3.8, $\left.\mu\right|_{\left\langle a, a^{y}\right\rangle}$ and $\left.\mu\right|_{\left\langle y, y^{a}\right\rangle}$ are g-nilpotent of class 2. Since $\left\langle a^{2},\left(a^{2}\right)^{y^{2}}\right\rangle=$ $\left\langle a^{2},\left(a^{y^{2}}\right)^{2}\right\rangle$ by Theorem [3.8, $\left.\mu\right|_{\left\langle a^{2},\left(a^{y^{2}}\right)^{2}\right\rangle}$ is g-nilpotent of class at most 2. Then by Theorem 3.7, $\left.\mu\right|_{\left.<a, a^{y^{2}}\right\rangle}$ is g-nilpotent of class at most 2. Similarly, since $\left\langle y^{3},\left(y^{3}\right)^{a^{3}}\right\rangle=\left\langle y^{3},\left(y^{a^{3}}\right)^{3}\right\rangle$ we have $\left.\mu\right|_{<y, y^{a^{3}}>}$ is g-nilpotent of class at most 2 . 
Now, we are ready to give some partial answer to the following question, which is fuzzy analogues of a main question on Engel groups. In the following we investigate the relation between torsion free $n$-Engel fuzzy subgroup and g-nilpotent fuzzy subgroups.

Theorem 3.10. Let $\mu$ be a normal torsion free 4-Engel fuzzy subgroup of $G$ and $a, y$ be conjugate elements of $G$. Then $\left.\mu\right|_{\langle a, y\rangle}$ is g-nilpotent of class at most 4 .

Proof. First we show that if $\mu(b)=\mu(e)$ and $\mu\left[a,{ }_{2} c\right]=\mu(e)$ for any $a, b, c \in G$, then

$$
\mu[a b, 2 c]=\mu(e), \quad(I)
$$

By Theorem 2.4 and since $\mu$ is normal, we have

$$
\begin{aligned}
\mu\left[a b,_{2} c\right] & \geq \mu\left(\left[[a, c]^{b}[b, c], c\right]\right) \geq \mu\left(\left[[a, c]^{b}, c\right]\right) \wedge \mu\left(\left[b,{ }_{2} c\right]\right) \geq \mu\left(\left[[a, c]^{b}, c\right]\right) \wedge \mu(b) \\
& =\mu\left(\left[[a, c]^{b}, c\right]\right)=\mu([[a, c][a, c, b], c]) \\
& \geq \mu\left([[a, c], c]^{[a, c, b]}\right) \wedge \mu([a, c, b, c])=\mu(e) \wedge \mu([a, c, b, c]) \\
& \geq \mu([a, c, b])=\mu\left([a, c]^{-1} b^{-1}[a, c] b\right) \\
& =\mu\left(b^{-[a, c]} b\right) \geq \mu(b) \\
& =\mu(e) .
\end{aligned}
$$

Hence $[a b, 2 c]=\mu(c)$. Now, by using Theorem 3.8 and since $[a, y]=a^{-1} a^{y}$ we have $\mu\left[x_{1}, x_{2}, x_{3}\right]=\mu(e)$ for any $x_{i} \in\langle[a, y], a\rangle$ which implies that $\left[a^{3}, y\right]=\left([a, y]^{a}\right)^{3} u ; \mu(u)=\mu(e)$, $(I I)$. Since $\left\langle\left[a^{3}, y\right], y\right\rangle=\left\langle y, y^{a^{3}}\right\rangle$, by Theorem [3.9, we have $\left.\mu\right|_{\left\langle\left[a^{3}, y\right], y\right\rangle}$ is g-nilpotent of class at most 2, which by (II) implies that $\left.\mu\right|_{\left\langle\left([a, y]^{a}\right)^{3}, y\right\rangle}$ is g-nilpotent of class at most 2 and so by Theorem [3.7, we have $\left.\mu\right|_{\left\langle[a, y]^{a}, y\right\rangle}$ is g-nilpotent of class at most 2. Hence $\mu\left[[a, y]^{a}, y, y\right]=\mu(e)$ and so

$$
\begin{aligned}
\mu\left[[a, y]^{a}, y, y\right]^{a^{-1}} & =\mu(e) \\
& \Longrightarrow \mu\left[\left[[a, y], y\left[y, a^{-1}\right]\right], y^{a^{-1}}\right]=\mu(e) \\
& \Longrightarrow \mu\left[\left[[a, y],\left[y, a^{-1}\right]\right][a, y, y]^{\left[y, a^{-1}\right]}, y^{a^{-1}}\right]=\mu(e) \\
& \Longrightarrow \mu\left[\left[[a, y],[a, y]^{a^{-1}}\right][a, y, y]^{\left[y, a^{-1}\right]}, y^{a^{-1}}\right]=\mu(e) \\
& \Longrightarrow \mu\left[\left[[a, y],[a, y]\left[a, y, a^{-1}\right]\right][a, y, y]^{\left[y, a^{-1}\right]}, y^{a^{-1}}\right]=\mu(e) .
\end{aligned}
$$

By considering $X=\left[[a, y],[a, y]\left[a, y, a^{-1}\right]\right]$ and $Z=[a, y, y]^{\left[y, a^{-1}\right]}$, we have

$$
\mu(e)=\mu\left(\left[X Z, y^{a^{-1}}\right]\right)=\mu\left(\left[X, y^{a^{-1}}\right]^{Z}\left[Z, y^{a^{-1}}\right]\right),
$$


If $\mu\left(\left[Z, y^{a^{-1}}\right]\right) \neq \mu(e)$, since

$$
\begin{aligned}
\mu\left(\left[X, y^{a^{-1}}\right]\right) & \geq \mu\left(\left[[a, y],[a, y]\left[a, y, a^{-1}\right]\right]\right) \\
& \left.=\mu\left(\left[[a, y],\left[a, y, a^{-1}\right]\right]\right)\right) \\
& =\mu\left(\left[\left[a, y, a^{-1}\right],[a, y]\right]\right) \\
& =\mu\left(\left[[a, y], a^{-1},[a, y]\right]\right) \\
& =\mu(e)(* * *)\left(\text { since }\left.\mu\right|_{\langle[a, y], a\rangle}\right. \text { is nilpotent of class 2) }
\end{aligned}
$$

then by Theorem [2.8, we have

$$
\begin{aligned}
\mu(e) & =\mu\left(\left[X, y^{a^{-1}}\right]^{Z}\left[Z, y^{a^{-1}}\right]\right) \\
& =\mu\left(\left[X, y^{a^{-1}}\right]^{Z}\right) \wedge \mu\left(\left[Z, y^{a^{-1}}\right]\right) \\
& =\mu(e) \wedge \mu\left(\left[Z, y^{a^{-1}}\right]\right) \\
& =\mu\left(\left[Z, y^{a^{-1}}\right]\right) .
\end{aligned}
$$

which is a contradiction. Therefore, $\mu(e)=\mu\left(\left[Z, y^{a^{-1}}\right]\right)$. Thus,

$$
\begin{aligned}
\mu(e) & =\mu\left(\left[Z, y a^{-1}\right]\right) \\
& =\mu\left(\left[[a, y, y]^{\left[y, a^{-1}\right]}, y^{a^{-1}}\right]\right) \\
& =\mu\left(\left[[a, y, y]^{y^{-1} a y a^{-1}}, y^{a^{-1}}\right]\right) \\
& \left.=\mu\left(\left[[a, y, y]^{y^{-1} a},\left(y^{a^{-1}}\right)^{(} y a^{-1}\right)^{-1}\right]^{y a^{-1}}\right) \\
& =\mu\left(\left[[a, y, y]^{y^{-1} a}, y\right]\right) \\
& =\mu\left(\left[\left([a, y, y]^{\left.y^{-1}\right) a}, y\right]\right)\right. \\
& =\mu\left(\left[\left([a, y, y]\left[a, y, y, y^{-1}\right]\right)^{a}, y\right]\right) \\
& =\mu\left(\left[[a, y, y]^{a}\left[a, y, y, y^{-1}\right]^{a}, y\right]\right) \\
& =\mu\left(\left[[a, y, y]^{a}, y\right]^{\left[a, y, y, y^{-1}\right]^{a}}\left[\left[a, y, y, y^{-1}\right]^{a}, y\right]\right) \quad(I I I) .
\end{aligned}
$$

Now, by Theorem [..9, $\left.\mu\right|_{\left\langle y, y^{a}\right\rangle}$ is g-nilpotent of class 2. Thus

$$
\mu\left[a, y, y, y^{-1}\right]=\mu\left(\left[y^{-a} y, y, y^{-1}\right]\right)=\mu(e), \quad(I V)
$$

which implies that $\mu\left(\left[\left[a, y, y, y^{-1}\right]^{a}, y\right]\right) \geq \mu\left(\left[a, y, y, y^{-1}\right]\right)=\mu(e)$ and so for the case $\mu(e) \neq$ $\mu\left(\left[[a, y, y]^{a}, y\right]\right.$ by Theorem $[2.8$ and (III) we have

$$
\mu(e)=\mu\left(\left[[a, y, y]^{a}, y\right]\right) \wedge \mu\left(\left[\left[a, y, y, y^{-1}\right]^{a}, y\right]\right)=\mu\left(\left[[a, y, y]^{a}, y\right]\right)
$$

which is a contradiction. Therefore

$$
\mu\left(\left[[a, y, y]^{a}, y\right]\right)=\mu(e), \quad(* * * *)
$$


By (IV), Theorem [2.4 and since $\mu$ is normal, we have

$$
\mu\left[[a, y, y]^{-1}, y\right]=\mu[a, y, y, y]^{-[a, y, y]^{-1}}=\mu(e)
$$

Then $\mu[[a, y, y], y]=\mu(e)$ and so

$$
\begin{aligned}
\mu[[a, y, y, a], y] & =\mu\left(\left[[a, y, y]^{-1}[a, y, y]^{a}, y\right]\right) \\
& =\mu\left(\left[[a, y, y]^{-1}, y\right]^{[a, y, y]^{a}}\left[[a, y, y]^{a}, y\right]\right) \\
& \geq \mu\left(\left[[a, y, y]^{-1}, y\right]\right) \wedge \mu\left(\left[[a, y, y]^{a}, y\right]\right) \\
& =\mu(e) \wedge \mu\left(\left[[a, y, y]^{a}, y\right]\right) \\
& =\mu(e) \quad(b y \quad(* * * *),
\end{aligned}
$$

Thus $\mu[[a, y, y, a], y]=\mu(e),(V)$. By Theorem [2.4, since $\left.\mu\right|_{\left\langle y^{a}, y\right\rangle}$ is nilpotent of class 2,

$$
\mu\left[[a, y, y, a], y^{a}\right] \geq \mu\left[[a, y, y], y^{a}\right] \wedge \mu\left[[a, y, y]^{a}, y^{a}\right]=\mu(e)
$$

which implies that

$$
\mu(e)=\mu\left(\left[a, y, y, a, y^{a}\right]=\mu([[a, y, y, a], y[y, a]])=\mu\left(\left[[a, y, y, a,[y, a]][a, y, y, a, y]^{[y, a]}\right) .\right.\right.
$$

If $\mu([[a, y, y, a,[y, a]]) \neq \mu([a, y, y, a, y])=\mu(e)($ See V), then by Theorem [2.8, we have

$$
\mu(e)=\mu([[a, y, y, a,[y, a]]) \wedge \mu([a, y, y, a, y])=\mu([[a, y, y, a,[y, a]])
$$

which is a contradiction. Thus, $\mu\left([[a, y, y, a,[y, a]])=\mu(e) \quad(V I)\right.$. Now, since $\left\langle\left[a, y^{2}\right], a\right\rangle=$ $\left\langle a^{y^{2}}, a\right\rangle$ by Theorem [3.9, we have $\left.\mu\right|_{\left\langle\left[a, y^{2}\right], a\right\rangle}$ is g-nilpotent of class at most 2 . Then by Theorem [2.4,

$$
\mu\left[[a, y]^{2}[a, y, y], a, a\right]=\mu\left(\left[[a, y][a, y]^{y}, a, a\right]\right)=\mu\left(\left[a, y^{2}, a, a\right]\right)=\mu(e)
$$

and since $\left.\mu\right|_{\left\langle y^{a}, y\right\rangle}$ is nilpotent, we get

$$
\mu\left[[a, y, y],[a, y]^{2}\right] \geq \mu\left[y^{-a} y, y, y^{-a} y y^{-a} y\right]=\mu(e)
$$

and since $x y=y x[y, x]$ for any $x, y \in G$, by

$$
[a, y, y][a, y]^{2}=[a, y]^{2}[a, y, y]\left[[a, y, y],[a, y]^{2}\right]
$$

and (I), we have

$$
\mu(e)=\mu\left(\left[[a, y, y][a, y]^{2}{ }_{2} a\right]\right)
$$

, where we put $a=[a, y]^{2}[a, y, y], b=\left[[a, y, y],[a, y]^{2}\right]$ in (I). Hence by Theorem [2.4,

$$
\mu(e)=\mu\left(\left[[a, y, y][a, y]^{2}{ }_{2} a\right]\right)=\mu\left(\left[[a, y, y, a]^{[a, y]^{2}}\left[[a, y]^{2}, a\right], a\right]\right)
$$

Now if $\mu\left(\left[[a, y, y, a]^{[a, y]^{2}}, a\right] \neq \mu\left(\left[[a, y]^{2}, a\right], a\right]\right)=\mu(e)$, then by Theorem [2.8, we have

$$
\left.\mu(e)=\mu\left(\left[[a, y, y, a]^{[a, y]^{2}}, a\right]\right) \wedge \mu\left(\left[[a, y]^{2}, a\right], a\right]\right)=\mu\left(\left[[a, y, y, a]^{[a, y]^{2}}, a\right]\right)
$$


which is a contradiction. Thus $\mu(e)=\mu\left(\left[[a, y, y, a]^{[a, y]^{2}}, a\right]\right)$. Hence by Theorem [2.4,

$$
\begin{aligned}
\mu(e) & =\mu\left(\left[[a, y, y, a]^{[a, y]^{2}}, a\right]\right) \\
& =\mu\left(\left[[a, y, y, a]\left[a, y, y, a,[a, y]^{2}\right], a\right]\right) \quad\left(\text { since } x^{y}=x[x, y] \text { for any } x, y \in G\right) \\
& =\mu\left([a, y, y, a, a]^{\left[a, y, y, a,[a, y]^{2}\right]}\left[a, y, y, a,[a, y]^{2}, a\right]\right) \quad(V I I)
\end{aligned}
$$

If $\mu([a, y, y, a, a])=\mu\left(\left[a, y, y, a,[a, y]^{2}, a\right]\right)$, since by (VI)

$$
\mu\left(\left[a, y, y, a,[a, y]^{2}, a\right]\right) \geq \mu\left(\left[a, y, y, a,[a, y]^{2}\right] \geq \mu([a, y, y, a],[a, y])=\mu(e)\right.
$$

we get $\mu[a, y, y, a,, a]=\mu(e)$. For the case $\mu([a, y, y, a, a]) \neq \mu\left(\left[a, y, y, a,[a, y]^{2}, a\right]\right)$, by Theorem 2.8 and (VII), we have

$$
\begin{aligned}
\mu(e) & =\mu\left([a, y, y, a, a]^{\left[a, y, y, a,[a, y]^{2}\right]}\right) \wedge \mu\left(\left[a, y, y, a,[a, y]^{2}, a\right]\right) \\
& =\mu\left([a, y, y, a, a]^{\left[a, y, y, a,[a, y]^{2}\right]}\right) \wedge \mu(e)=\mu\left([a, y, y, a, a]^{\left[a, y, y, a,[a, y]^{2}\right]}\right) \\
& =\mu[a, y, y, a, a]
\end{aligned}
$$

which is a contradiction. Hence $\mu[a, y, y, a, a]=\mu(e)$ which by $(\mathrm{V})$ implies that, $[a, y, y, a] \in$ $Z\left(\left.\mu\right|_{\langle a, y\rangle}\right)$. Now, by (IV) and Lemma [2.], $[a, y, y] \in Z_{2}\left(\left.\mu\right|_{\langle a, y\rangle}\right)$. Similarly $[y, a, a] \in$ $Z_{2}\left(\left.\mu\right|_{\langle a, y\rangle}\right)$ and so $[y, a] \in Z_{3}\left(\left.\mu\right|_{\langle a, y\rangle}\right)$. Therefore, by Lemma [2], $\left.\mu\right|_{\langle a, y\rangle}$ is a nilpotent of class at most 4 .

Corollary 3.11. Let $\mu$ be a normal torsion free 4-Engel fuzzy subgroup of $G$. Then for any $x, z \in G,\left.\mu\right|_{\langle x z, z y\rangle}$ is g-nilpotent of class at most 4 .

Proof. By Theorem B.J0, $\left.\mu\right|_{\langle a, y\rangle}$ is g-nilpotent of class at most 4 in which $a, y$ are conjugate elements of $G$. Therefore, $\left.\mu\right|_{\langle x z, z x\rangle}=\left.\mu\right|_{\left\langle x z, x^{-1}(x z) x\right\rangle}$ is g-nilpotent of class at most 4 .

\section{Conclusions}

The study of Engel fuzzy subgroups allowed us to solve existence problems on g-nilpotent fuzzy subgroups and so on nilpotent groups. In this paper we can justify our definition of a g-nilpotent fuzzy subgroup. In the classical group theory there is an open question: Is every torsion free $n$-Engel group (for $n \geq 4$ ), nilpotent?. To answer the question, Traustason [II] showed that with some additional conditions all 4-Engel groups are locally nilpotent. Here, we gave some partial answer to this question on Engel fuzzy subgroups. Naturally, the results attained prompt us for further researches on the subject.

\section{Acknowledgments}

The authors wish to sincerely thank the referees for several useful comments. 


\section{REFERENCES}

[1] R. Ameri, R.A. Borzooei, E. Mohammadzadeh, Engel fuzzy subgroups, Italian Journal of Pure and Applied Mathemetics, 34 (2015), pp. 251-262.

[2] R.A. Borzooei, M. Bakhshi, T-fuzzy congruences and T-fuzzy filters of a BL-algebra, Iranian Journal of Fuzzy Systems, 6(4) (2009), pp. 37-47.

[3] R.A. Borzooei, E. Mohammadzadeh, V. Fotea, On Engel Fuzzy Subpolygroups, New Mathematics and Natural Computation, to appear.

[4] E.S. Golod, Some problems of Burnside type, Proc. Int. Congr. Math., Moscow (1966), pp. 284-289.

[5] H. Heineken, Engelsche Elemente der Lange drei, Illinois Journal of Mathematics, 5 ( 1961), pp. 681-707.

[6] L.C. Kappe and W.P. Kappe, On three-Engle groups, Bulletin of the Australian Mathematical Society, 7(3) (1972), pp. 391-405.

[7] F.W. Levi, Groups in which the commutator operation satiesfies certain algebric conditions, Journal of the Indian Mathematical Society, 6 (1942), pp. 87-97.

[8] J.N. Mordeson, K.R. Bhutani, A. Rosenfeld, Fuzzy group theory, Springer (2005).

[9] D. Robinson, A course in the theory of groups, Springer (2012).

[10] A. Rosenfeld, Fuzzy groups, Journal of Mathematical Analysis and Applications, 35(3) (1971), pp. $512-517$.

[11] G.Traustason, On 4-Engle groups, Journal of Algebra, 178(2) (1995), pp. 414-429.

[12] J. Wiegold, Transitivegroups with fixed-point-free permutations, Archiv der Mathematik, 27 (1976), pp. 473-475.

[13] J. Wiegold, Transitivegroups with fixed-point-free permutations II, Archiv der Mathematik, 29 (1977), pp. 571-573.

[14] L.A. Zadeh, Fuzzy sets, Information and Control, 8 (1965), 338-353.

[15] M. Zorn, Nilpotency of finite groups, Bulletin of the American Mathematical Society, 42 (1936), pp. 485-486.

[16] A. Abdollahi, S. Janbaz and M. Oubodi, Graphs Cospectral with A Friendship Graph Or its Complement, Trans. Combin. 2(4) (2013), pp. 37-52.

[17] N.L. Biggs, Algebraic Graph Theory, (second edition), Cambridge University press, cambridge, (1933).

\section{E. Mohammadzadeh}

Department of mathematics

Payame Noor University

P.O. Box 19395-3697

Tehran, Iran.

mohamadzadeh36@gmail.com

\section{R. A. Borzooei}

Department of mathematics

Shahid Beheshti University, G. C.,

Tehran, Iran.

borzooei@sbu.ac.ir 


\section{Young Bae Jun}

Department of mathematics Education

Gyeongsang National University

Jinju 52828, Korea.

skywine@gmail.com 